\title{
Strahlmaschinen virtuell optimieren
}

\author{
Mit einer speziellen Simulationssoftware lässt sich der Bearbeitungsprozess in der Strahlmaschine \\ innerhalb weniger Minuten visualisieren und optimieren. Dadurch können Anlagen schnell und \\ flexibel auf die individuellen Anforderungen der Betreiber zugeschnitten werden.
}

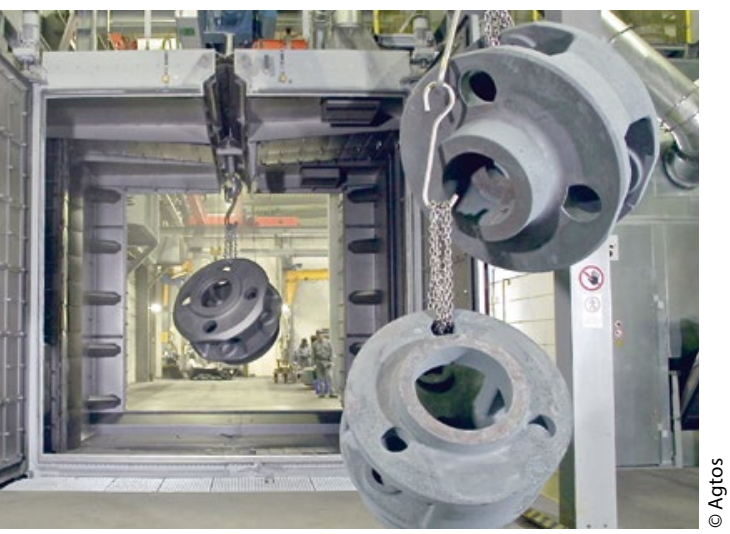

Bild 1 > Die neue Software hilft bei Auslegung und Einstellung der Strahlmaschinen.

Auf den ersten Blick haben Motorblöcke, Stahlträger, Kochtöpfe und Zahnräder wenig gemeinsam. Es existieren jedoch Parallelen: Alle haben metallische Oberflächen, die mit Schleuderrad-Strahlmaschinen bearbeitet werden. Dabei katapultiert ein rotierendes Schleuderrad, das sich an exakt definierten Außenpositionen der Anlagen befindet, metallisches Strahlmittel (Stahlguss, Hartguss, Aluminium, Edelstahl) oder nicht metallisches Strahl- mittel (Korunt, Keramik, Schlacke) auf die Werkstücke. Mit dieser Technik lassen sich Oberflächen entzundern, entrosten, entschichten sowie entgraten, aufrauen oder glätten. Sogar ein Verfestigen beziehungsweise Umformen ist möglich. Die Bearbeitung der Oberflächen in den Strahlmaschinen ist ein komplexer Vorgang. Das Strahlgut muss im richtigen Winkel und mit der richtigen Energie auftreffen. Daher ist eine gewisse Erfahrung nötig, um die Parameter so einzustellen, dass der Betreiber mit einer Anlage exakt das gewünschte Ergebnis erzielt.

\section{Simulation anhand der CAD-Daten des Werkstücks}

Eine neue Software unterstützt Techniker und Ingenieure bei Agtos nun bei der Auslegung der Maschinen. Entwickelt wurde die sogenannte DRS-Simulationssoftware am Fraunhofer-Institut für Produktionstechnik und Automatisierung IPA in Stuttgart. DRS steht dabei für Dynamische Reinigungssimulation.
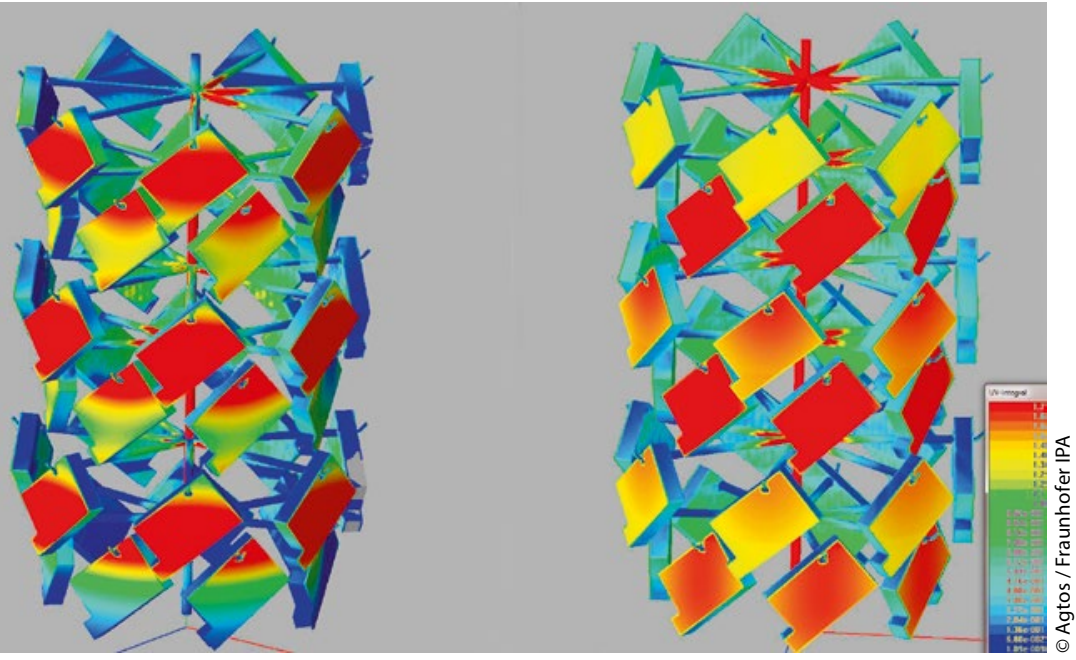

Zunächst wurde hierfür eine Machbarkeitsstudie durchgeführt, in der das Programm an praktischen Beispielen - darunter die Bearbeitung von Motorblöcken, Stahlträgern, Kochtöpfen und Zahnrädern - getestet wurde. Mittlerweile ist die Simulationssoftware bei dem Maschinenbauer routinemäßig im Einsatz.

Mussten die Techniker bisher die richtige Auslegung und Einstellung einer Maschine mit Hilfe von Original-Werkstücken des Betreibers experimentell ermitteln, so reicht in vielen Fällen nun ein Maus-Klick. Die Simulations-Software ist intuitiv bedienbar und benötigt lediglich die CAD-Daten des Werkstücks, das bearbeitet werden soll.

Auf dem Monitor können die Techniker das virtuelle Bauteil in der Anlage platzieren, drehen, einzelne Streuräder aktivieren oder deaktivieren, das Strahlgut wählen, den Abstand zwischen Schleuderrad und Werkstück verändern und einen kompletten Durchlauf starten. Final visualisiert das Programm den Energieeintrag; bilden sich starke Schatten ist eine gleichmäßige Bearbeitung nicht möglich (Bild 2). Mithilfe der neuen Software können Anlagen schneller und flexibler auf die individuellen Anforderungen der Betreiber zugeschnitten werden. //

\section{Kontakt}

Fraunhofer-Institut für Produktionstechnik und Automatisierung IPA, Stuttgart Jörg Schieweck

Tel. 07119701874

joerg.schieweck@ipa.fraunhofer.de www.ipa.fraunhofer.de 